Non- cont act and real-ti ne measur ement of heart $r$ ate and heart $r$ ate variabi I ity using mi cr owave r ef I ect omet $r y$

\begin{tabular}{|l|l|}
\hline 著者 & $\begin{array}{l}\text { MASE At sushi, KOGA Y., MARUYANA T. , TOKUZAWA } \\
\text { Toki hi ko, SAKAI F., KUNUG TA M, KOI KE T. , } \\
\text { HASEGAMA H. }\end{array}$ \\
\hline 杂譙志名 & Revi ew of Sci ence I nst r ument s \\
\hline 巻 & 91 \\
\hline 号 & 1 \\
\hline ページ & 014704 \\
\hline 発行年 & 2020 01 \\
\hline URL & ht t p: //hdl . handl e. net /10655/00012833 \\
\hline
\end{tabular}

doi: $10.1063 / 1.5128959$ 


\title{
Non-contact and real-time measurement of heart rate and heart rate variability using microwave reflectometry
}

\author{
A. Mase, ${ }^{1,2, a)}$ Y. Kogi, ${ }^{3}$ T. Maruyama, ${ }^{4,}{ }^{5}$ T. Tokuzawa, ${ }^{6}$ F. Sakai, ${ }^{7}$ M. Kunugita, ${ }^{8}$ T. Koike, ${ }^{8}$ and H. Hasegawa ${ }^{8}$ \\ 1) Global Innovation Center, Kyushu University, Kasuga 816-8580, Japan \\ 2) Electronics Research Laboratory, Fukuoka Institute of Technology, Fukuoka 811-0295, Japan \\ 3) Department of Information Electronics, Fukuoka Institute of Technology, Fukuoka 811-0295, Japan \\ 4) Faculty of Art \& Science, Kyushu University, Fukuoka 819-0395, Japan \\ ${ }^{5)}$ Kyushu University Hospital, Fukuoka 812-8582, Japan \\ 6) National Institute for Fusion Science, Toki 509-5292, Japan \\ 7) Sakura Tech Co., Yokohama 222-0033, Japan \\ ${ }^{8)}$ Tokai Rika, Co. Ltd., Oguchi 480-0195, Japan
}

(Received:

\begin{abstract}
In this paper we present non-contact and non-invasive vital signal detection using microwave reflectometer. Elimination of noise components due to random movement of human subjects has been the biggest issue for microwave measurement. Appropriate filtering, amplitude control of reflectometer signal, and cross-correlation among multiple reflectometers together with new algorithms have enabled motion artifact elimination, signal peak detection, and data processing for various parameters related to heart rate (HR) and heart rate variability (HRV). We focus here on the real time measurements of instantaneous HR and HRV for the practical use. The evaluation using microwave reflectometry is completely noninvasive and feasible even through clothing, which is extremely effective for health maintenance in daily life as well as for preventing sudden death related to, for example, coronary heart disease and ventricular arrhythmia.
\end{abstract}

\section{INTRODUCTION}

It is said that heart disease and stroke mortality rates have stopped declining although the mortality rates due to other causes globally decrease. ${ }^{1,2}$ Health monitoring and healthcare are becoming increasingly important. Heart rate (HR) and its fluctuation (heart rate variability: HRV) are very useful parameters for such monitoring. ${ }^{3-7}$ Several methods have been developed for the measurement. Those are conventional electrocardiograph (ECG), piezo-electric sensor, ultrasonic sensor, infrared sensor, and microwave reflectometry sensor. Recently, light and body color changes have been utilized as a remote measurement of HR and HRV by Rochester Group. ${ }^{8}$ Non-contact and non-invasive diagnostic method should be very useful since it is applied to any type of subject without giving an uncomfortable feeling. Note that the detection of arrhythmia (atrial fibrillation, in particular) by non-contact method is an important issue to prevent serious heart disease. Microwave reflectometry measures remotely the human skin and/or internal organ movements when breathing and heartbeat are present rather than the electrical impulses usually measured by ECG. ${ }^{9-14}$

The heartbeat interval is constantly fluctuating by autonomous nerve activity comprised of sympathetic and parasympathetic nerve activity. The time variation can be regarded as the short-term time variation of the HR frequency, the so-called HRV. In the frequency spectrum of the HRV, the parasympathetic nerve activity appears in the spectral region of $0.15-0.45 \mathrm{~Hz}$ (the so called HF region), and both sympathetic and parasympathetic nerve activity appears in the region of $0.03-0.15 \mathrm{~Hz}$ (the so called LF region). ${ }^{15-20}$ The peak ratio or area ratio of the power spectrum in the LF component to the HF component is used to evaluate the stress condition. The sympathetic nerve activity increases in a stressful state and the parasympathetic nerve activity increases in a relaxed state. The ratio $\mathrm{LF} / \mathrm{HF}$ in a stressful state is larger than that in a relaxed

\footnotetext{
a) Electronic mail: mase@gic.kyushu-u.ac.jp
}

state. The coefficient of variation of R-R intervals (CVRR) can also be introduced as one of the indices to evaluate autonomous nerve activity. The value of CVRR is related to the standard deviation of $\mathrm{N}-\mathrm{N}$ interval ( $\mathrm{SDNN}$ ) as $\mathrm{CVRR}=\mathrm{SDNN} / \bar{x}$, where $\mathrm{N}-\mathrm{N}$ interval is equivalent to R-R interval and $\bar{x}$ is the mean value of $R-R$ interval.

Combining these indices (time variation of $\mathrm{HR}$ and CVRR and frequency spectra of HRV), the human mental and physical conditions can be evaluated with reliability. ${ }^{21,22}$ Conventional ECG monitoring is a major tool for evaluating HRV. However, it requires patients to be tethered to the monitoring devices, therefore, the evaluation method using ECG seems to be unsuitable as a long-time monitoring of subjects. Specifically, there is some concern regarding the practical application of the ECG system to sick persons and elderly persons. It is noted that the stress has a variety of forms including acute stress and chronic stress. There is various opposition in relation to the stress condition and the HRV indices. ${ }^{23-25}$ Further study should be necessary. In the present study, we would like to state that present microwave reflectometer could be utilized in replace of ECG.

Two novel elements have been introduced in relation to the signal processing for the HRV reconfiguration in microwave reflectometric measurements. Those are template/crosscorrelation algorithm and maximum entropy method (MEM) algorithm. ${ }^{26,27} \mathrm{We}$ introduce here a new algorithm for real-time evaluation of HR/HRV, and apply to subjects' measurements.

The contents of this paper are organized as follows: In Section II, the details of microwave reflectometry system including new developed one are described. The signal processing for real time evaluation of HR and HRV is studied in Section III. In Section IV, the experimental results of HR and HRV are demonstrated and followed by conclusions. The acronyms in the manuscript and their explanations are summarized in the nomenclature. 


\section{RADAR REFLECTOMETER FOR VITAL SIGNAL DETECTION}

The radar (radio detecting and ranging) techniques have been applied as a means to detect the distance and the direction to an object. Three types of radar systems are mainly utilized, those being: frequency-modulated continuous-wave (FM-CW) radar, pulse radar, and fixed-frequency Doppler radar. When the target position changes periodically, we can evaluate the fluctuation frequency from the radar output signals. This can be utilized for the measurement of vital signals as a non-contact method.

The most common method for vital signal detection will be a fixed-frequency reflectometer (Doppler radar), since its electronics circuit is rather simple and low cost as well as easy to be permitted as a consumer appliance under the Administration of Radio, the Ministry of Internal Affairs and Communications (MIC), Japan. ${ }^{28}$ The fixed frequency reflectometry shown in Fig. 1 utilizes a Doppler effect produced by the movement of a target. The beat frequency between the transmitted wave and the reflected wave gives the information of target movement. The distance to a target is given by $L+\delta L$

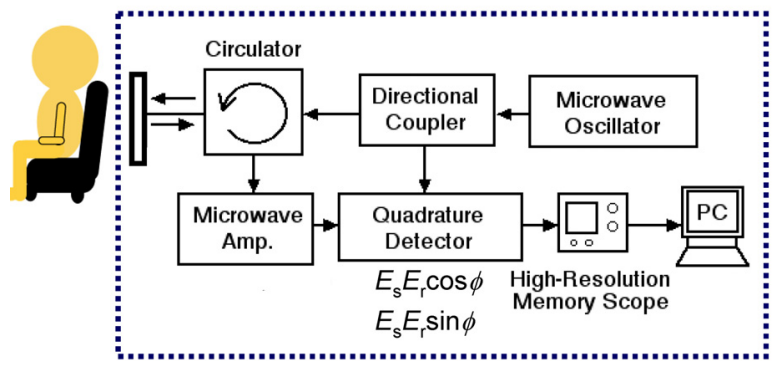

FIG. 1. Schematic of homodyne Doppler reflectometer.

when the target is moving with a fluctuation of $\delta L$ around the position $L$. The received signal from a target is given by

$$
E_{S}(t)=E_{s} \cos \{\omega t+2 k(L+\delta L)\}=E_{s} \cos \left(\omega t+\phi_{s}+\Delta \phi\right),
$$

where $\phi_{s}$ is the fixed phase without fluctuation. The low frequency component of the mixer output between reflected wave and reference wave is calculated as

$$
\begin{aligned}
& \overline{\left|E_{s} \cos \left(\omega t+\phi_{s}+\Delta \phi\right)+E_{r} \cos \left(\omega t+\phi_{r}\right)\right|^{2}} \\
& =\frac{1}{2}\left(E_{s}^{2}+E_{r}^{2}\right)+E_{s} E_{r} \cos \left(\phi_{0}+\Delta \phi\right),
\end{aligned}
$$

where upper bar denotes the time integration, $\phi_{r}$ is the initial phase of a reference wave, and $\phi_{0}=\phi_{s}-\phi_{r}$. The reflectometer signal includes the fluctuation component caused by the target position movement. When the target position fluctuates periodically, we can obtain the information of the frequency from the radar output. However, the target position $L$ cannot be identified by the fixed frequency reflectometer.

An FM-CW radar utilizes a frequency-swept oscillator, such as voltage-controlled oscillator (VCO) instead of a fixedfrequency oscillator. The output frequency of a VCO is varied by changing the magnitude of an input voltage, such as sawtooth-wave voltage. The reflected wave from a target suffers phase delay or group delay depending on the distance to the target as shown in Fig. 2. Therefore, the mixer output has an intermediate frequency $\left(f_{I F}\right)$ given by

$$
f_{I F}=(\Delta f / \Delta t) \tau=B \tau / T_{m}=2 B L f_{m} / c,
$$

where $T_{m}$ is the period of sawtooth wave, $B$ is the frequency bandwidth, and $\tau=2 L / c$. The distance to the target is evaluated by performing Fourier analysis of the mixer output signal. The output frequency is fluctuating around the intermediate frequency $\left(f_{I F}\right)$ depending on the target position movement $\delta L$ caused by the vital signals.

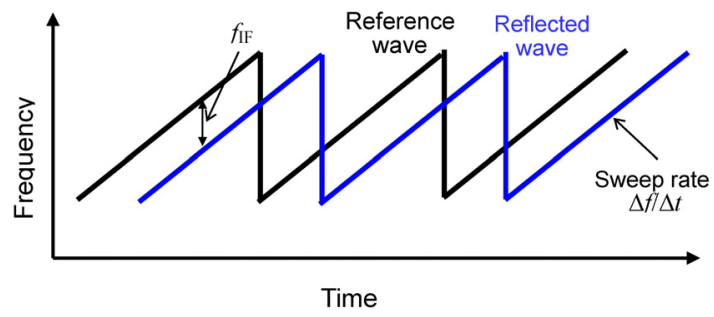

FIG. 2. Relationship between reflected wave and reference wave.

On the other hand, a pulse radar transmits an intermittent signal with alternate on- and off-periods. The waveform of an output wave is described by an amplitude modulated fixed frequency wave. The reflected wave from a target experiences a round trip delay time of $\tau=2(L+\delta L) / c$. Compared to a fixed frequency reflectometer, both an FM-CW radar and a pulse radar can identify the target position $L$. The information of the target position gives an advantage if there are plural subjects to be measured, since the signals obtained from each subject can be identified. ${ }^{29,30}$

The frequencies of 10.5-10.55 GHz and 24.05-24.25 GHz are approved as specific small electricity radio station for movement body detection sensors from MIC. ${ }^{28}$ Therefore, it is rather easy to obtain modules in the frequency range. In the present experiments, $24 \mathrm{GHz}$ radar modules (JRC NJR4265J) have been applied first to the measurements, since outdoor use of the $24 \mathrm{GHz}$ modules is authorized in Japan as a movement body detection sensor when the radiation power is low. ${ }^{28}$ The modules have I-Q terminals. Each signal is inputted to AD converter to obtain the phase fluctuation due to vital signals.

In a $10 \mathrm{GHz}$ system, a heterodyne reflectometer using an automatic gain control (AGC) amplifier is utilized to control the reflected wave. ${ }^{31,32}$ We have now fabricated a new $24 \mathrm{GHz}$ homodyne reflectometer as shown in Fig. 3. A microwave oscillator is used as both an incident wave and a local oscillator (LO) wave. One of the waves is irradiated onto the area near the human heart or the path of an artery in the thigh via a single patch antenna or an array antenna. The directivity of antennas must be selected depending on the position of the measurement. High gain antennas are needed in the case of remote measurement $(>1 \mathrm{~m})$. However, in the present experiment, rather low gain antennas are utilized for the short distance measurement ( $\leq 5 \mathrm{~cm}$, beam radius is $\sim 3 \mathrm{~cm}$ at the irradiation position). Since the phase fluctuation must be analyzed, the optical alignment of radiation beam is not important so long as a sufficient signal to noise ratio is observed. The reflected wave from the skin or the surface of the heart is picked up by an identical antenna, and amplified by a low noise microwave amplifier. A voltage-controlled attenuator is utilized to control the reflected wave in both static and non-static environments of 
a human subject. A good signal to noise ratio (SNR) for evaluation of HR/HRV is obtained using this system with an output power less than $-40 \mathrm{dBm}(100 \mathrm{nW})$, which satisfies the condition of weak radio station under the MIC, Japan. ${ }^{28}$

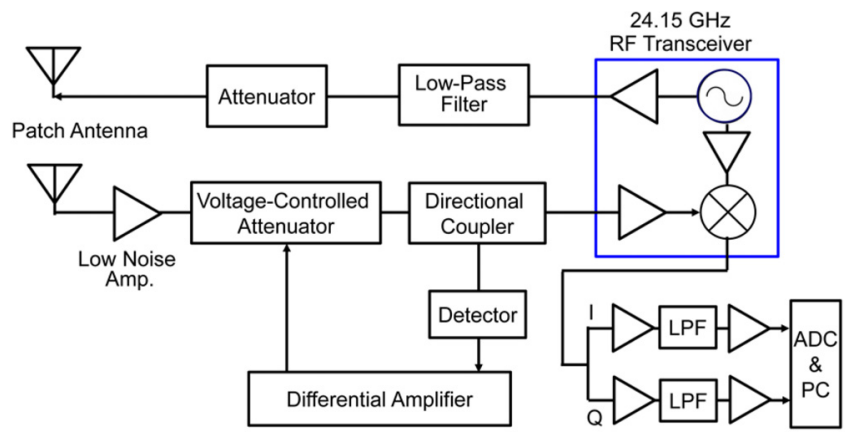

FIG. 3. Schematic of the new $24 \mathrm{GHz}$ system with an AGC attenuator.

\section{SIGNAL PROCESSING FOR HR/HRV MEASUREMENT}

\section{A. Elimination of spurious body movement}

Since the skin depth of electromagnetic waves is much smaller than a wavelength at the frequency larger than $10 \mathrm{GHz}$, an incident wave is mostly reflected at the skin layer of a target (human subject). The phase change of the reflectometer is caused by the skin movement synchronized with a heartbeat.

When a human subject is in a stationary state such as during sleeping, the main reflectometer signal includes heartbeat signal and respiration signal. An example of the signal waveform is shown in Fig. 4. Since each period (=1/frequency) of heartbeat and respiration signal is different, the separation of both components is performed by using appropriate filters. A matched filter could also be utilized in order to distinguish both components. $^{29}$

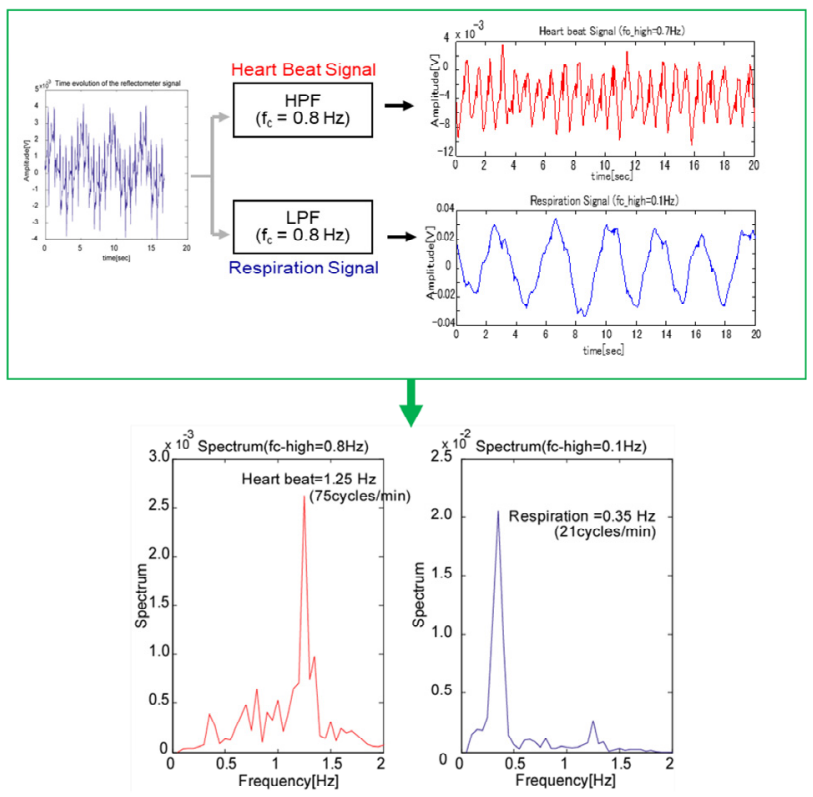

FIG. 4. (top) Waveform of the reflectometer signal and filtered signals. (bottom) Frequency spectra of each signal.

In a non-stationary state of a subject, the reflectometer signal includes random and large body movements. The movements often have a wide range of frequency including the region of heartbeat frequency. It is therefore difficult to distinguish the heartbeat signal. We have succeeded in reducing the noise component and retrieve the heartbeat signal by calculating a cross-correlation function between two measurement signals obtained from two reflectometers, $A(t)$ and $B(t)$ as given by

$$
E(t)=\lim _{T \rightarrow \infty} \frac{1}{T} \int_{-T / 2}^{T / 2} A(t) B(t+\tau)
$$

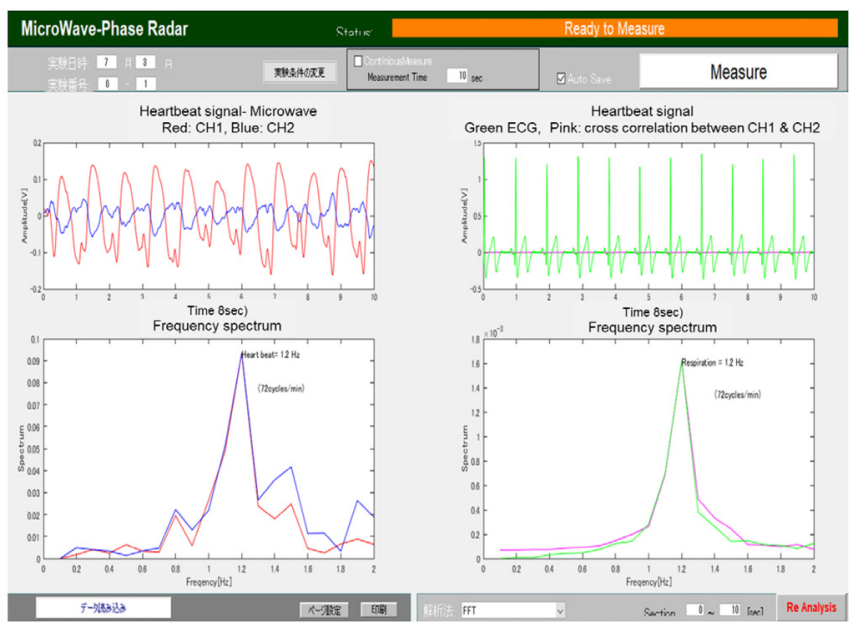

FIG. 5. (left column) Waveform and frequency spectra of the two reflectometers; (right column) Waveform of the ECG (green) and crosscorrelation between two microwave signals (pink).

The spurious noises of the radar reflectometers due to random body movement are cancelled by the above correlation. However, the heart beat components do not disappear. An example of the measurement is shown in Fig. 5, which shows an example of the measurement for a person sitting on a chair during his desk work together with ECG (Parama-Tech ECG recorder EP-202 with electrodes). Microwave reflectometers measure two positions under the thigh. Therefore, respiration signal and spurious components due to body movement are rather small compared to the measurement near the chest. The left column shows waveforms and frequency spectra of the two reflectometers. The right column shows waveforms and frequency spectra of the ECG and cross-correlation between two microwave signals. It is shown that the spurious noises of the radar reflectometers are cancelled by the above correlation, and the signal-to-noise ratio of the heart beat spectrum is improved.

\section{B. Algorithm for real time evaluation of instantaneous heart rate and heart rate variability}

In recent years, monitoring of heart-rate variability (HRV) has become of importance in order to prevent sudden death related to, such as, coronary heart disease and ventricular arrhythmia. The HRV evaluation is also recognized as an advanced application to prevent stress syndrome, detection of sleep prediction in driving a car, and other applications. The HRV is generally obtained from R-R intervals in an ECG. As was shown in the previous section, the microwave reflectometer signal includes various fluctuation noise due to random movement of the body surface as well as the respiration 
component, thus making it more difficult to detect the heartbeat interval corresponding to the R-R interval of ECG signals. As shown in Fig. 5, the detection of peak (R-R) intervals in an ECG signal is easy. However, it is rather difficult in a microwave signal to determine the peak interval precisely. The two methods (algorithms) are proposed to evaluate instantaneous heartbeat interval (heart rate). ${ }^{26,27}$

One is cross-correlation technique. In this algorithm, a template signal is formed by the arithmetic average of a reflected wave. Then, the cross-correlation function between the template and the measurement signal is calculated to obtain a clear peak waveform for evaluation of R-R intervals. The other is MEM algorithm. ${ }^{33}$ The time variation of the heartbeat frequency is evaluated by applying the MEM repeatedly over the short term. The time window is shifted step by step along the temporal axis. The HRV can then be reconstructed once the value of the heartbeat interval is calculated by the inverse of the heartbeat frequency.

When the radar reflectometer is applied to the measurement of vital signals in a daily life, for example, under sleeping, working, and driving, the real time evaluation of HR and HRV becomes extremely important. We have developed a new algorithm for real time evaluation of those parameters. One is to measure the time evolution of HR using time-to-frequency spectrum analysis (wavelet transform) and the other is to measure the instantaneous HR (the time evolution of R-R interval) using developed algorithms.

The overview flowchart of the proposed algorithm is shown in Fig. 6. The steps (procedures) are as follows: i) The various parameters (data width and measurement time, shift time and spectral analysis method for HRV) are chosen via a data logger (Graphtec GL980): ii) The data recording start: iii) If enough

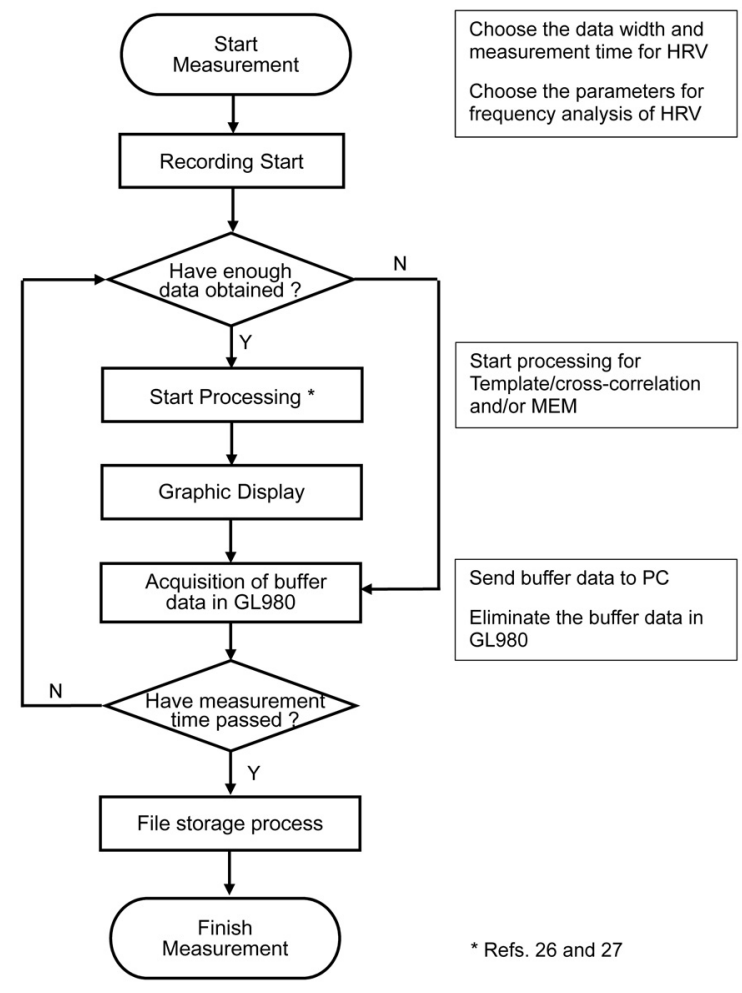

FIG. 6. Flow chart for real time HRV measurement. data is obtained, the HRV analysis using above algorithm starts, and various HRV indices are displayed. If not, the process returns to data recording. These steps are repeated following the shift time. The key point of the program is an application of parallel processing of data collection and data analysis. The data logger has $4 \mathrm{MW} / \mathrm{CH}$ RAM and 4 GB flash memory with 16 bit AD converter.

\section{EXPERIMENTAL RESULTS AND DISCUSSION}

\section{A. Long time measurement of HR}

The radar reflectometers have been applied to the overnight monitoring of HR. The waveguide antenna or substrate antenna (patch antenna) is installed in the back side of a bed, so that the microwave is irradiated onto the heart

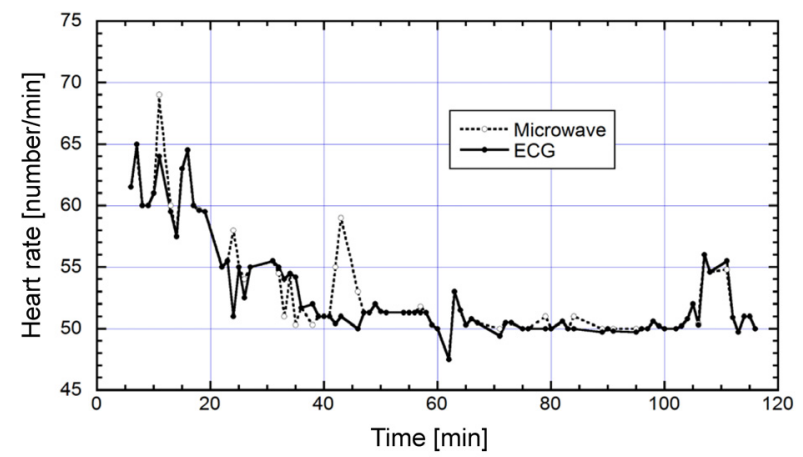

FIG. 7. Measurement of HR for a subject under sleeping.

region from the back of a human body. We use a high-pass filter with cutoff frequency of $0.8 \mathrm{~Hz}$ to distinguish a heartbeat signal from a respiration one.

In Fig. 7 is shown an example of HR measurement by using a microwave reflectometer and an ECG. The short time fast Fourier transform (FFT) is applied to obtain the time evolution of the HR. The time window size for short time FFT is $50 \mathrm{~s}$. The correlation coefficient between two values obtained by a radar reflectometer and an ECG is 0.97 .

\section{B. Real time measurement of HR and HRV}

The measurement and evaluation of HR and HRV are performed using the above mentioned algorithms. Since HRV is provided by plotting the peak (R-R) intervals as a function of time, it is frequency transformed by using FFT or MEM. As mentioned previously, parasympathetic nerve activity appears in the spectral region of $0.15-0.45 \mathrm{~Hz}$ (HF region), and both sympathetic and parasympathetic nerve activity appears in the region of $0.03-0.15 \mathrm{~Hz}$ (LF region). The peak ratio or area ratio of the power spectrum in the LF component to the HF component is used to evaluate the stress condition.

As shown in Refs. 31 and 32, a heterodyne-type reflectometer with frequency of $10 \mathrm{GHz}$ was utilized for the initial test. ${ }^{26}$ Large discrepancy of the HRV data often occurred between the original microwave reflectometer signal and the ECG signal, since large peaks due to the body motion will be picked up rather than the peaks due to heart beat without any data processing. The HRVs calculated by two proposed methods 
agreed well with the data measured by the ECG. The frequencies of the HRVs calculated by two proposed methods and the ECG were analyzed respectively. As shown in Ref. 26, the comparative results were presented for a relaxed state and a stressful state, respectively. The ratio of LF/HF obtained by two proposed methods were consistent in the range of $\pm 30 \%$ compared to that obtained by the ECG in each state.

The evaluation of LF/HF from HRV frequency spectra has been applied to 9 subjects under the various conditions. It was shown that there is a scattering in the LF/HF values depending on each individual, however, the tendency was quite similar. The LF/HF values obtained by microwave reflectometers were compared with those obtained by an ECG. The agreement seems to be good (the correlation coefficient $r=0.769$, the regression line $y=0.90504 x$ ) considering that the individual variability of $\mathrm{LF} / \mathrm{HF}$ is said to be about $\pm 30 \%$. $^{21}$

The $24 \mathrm{GHz}$ modules are being applied to the real time measurement of HR and evaluation of HRV. The tests are usually performed for subjects under working with a personal computer (PC). As a first step, the time evolution of heart rate spectra is obtained by using wavelet transform as shown in Fig. 8 . The analysis is performed in every fixed time interval $(5 \mathrm{sec}$ in this plot) and displayed on a PC continuously. Two microwave sensors are utilized in this measurement. The cross correlation between two sensors is also calculated. The difference of the peak values of spectra between microwave reflectometry and ECG signals (called error rate assuming the ECG value is correct) is shown in the top right trace. The error rate is less than a few present. A good agreement between microwave and ECG is obtained.

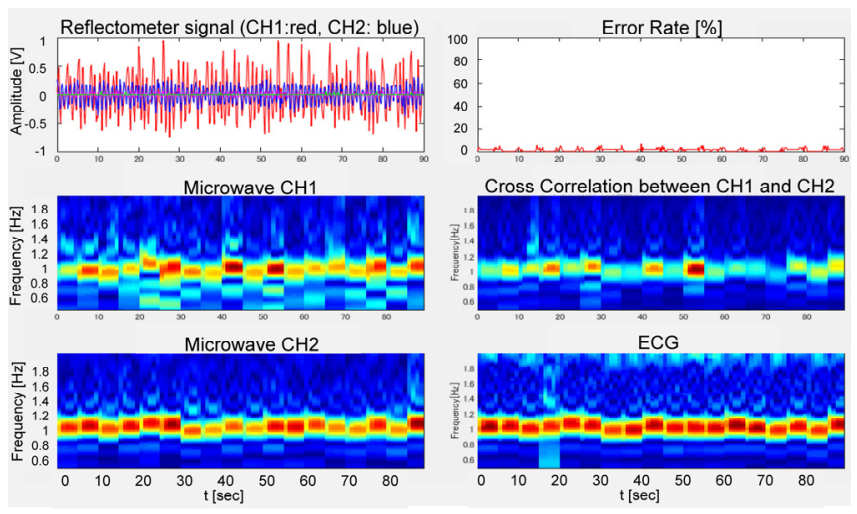

FIG. 8. Real-time measurement of HR spectra: (top left) waveforms of reflected signals, (top right) displacement rate of the peak value between microwave and ECG spectra, (middle and bottom) wavelet transform of two microwave sensors (CH1 and $\mathrm{CH} 2$ ), cross-correlation between $\mathrm{CH} 1$ and $\mathrm{CH} 2$, and ECG.

The time evolution of HRV indices as well as of HR obtained by both template/cross-correlation method and MEM method are shown in Fig. 9. The analysis time window for both algorithms is typically $30-40$ seconds, which is shifted in every 1-2 seconds. It is shown that the time evolutions of HR and CVRR obtained by reflectometry are in good agreement with those by ECG. The absolute value of LF/HF shows discrepancy, however, the tendency of time behavior is in agreement. Note that the present HRV measurements are limited to the short time ( $\sim 5$ min.) evaluation.

In Fig. 10, the physical stress (an ice test) ${ }^{32}$ is applied to a subject during a time interval shown in the figure. An ice test is applied to a subject. It is seen that the values of $\mathrm{HR}$ and $\mathrm{LF} / \mathrm{HF}$ increase during the stress conditions.
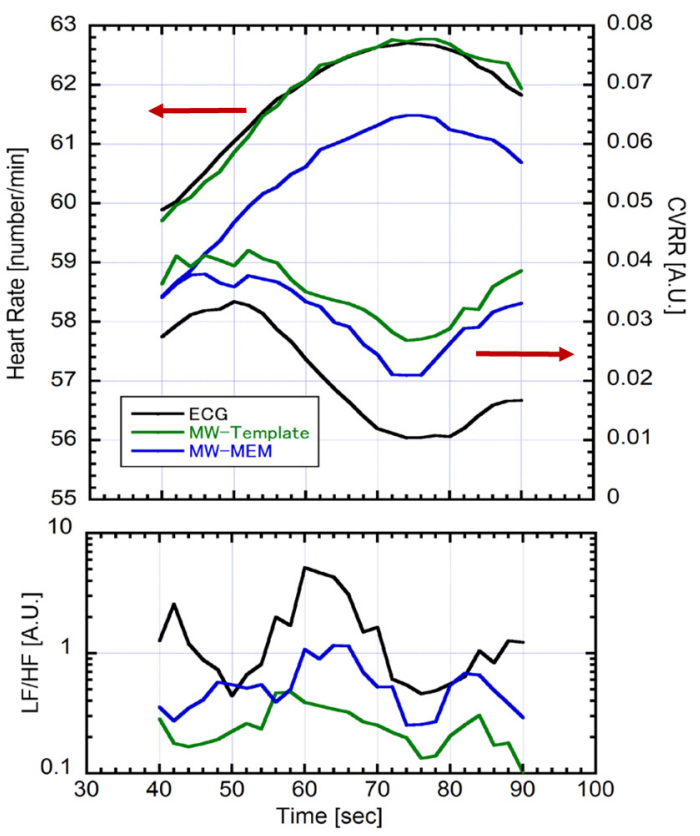

FIG. 9. Comparison of microwave and ECG signals for HR and HRV indices; (black) ECG, (green) Template/cross-correlation, (blue) MEM.

We can summarize the behaviors of experimental results of $\mathrm{HR}$ and $\mathrm{LF} / \mathrm{HF}$ as:

i) The change rate of HR is usually less than $5 \%$ in a normal condition without stress. However, it increases up to $10 \%$ or more in the stressful state.

ii) The value of $L F / H F$ increase in a stressful state.
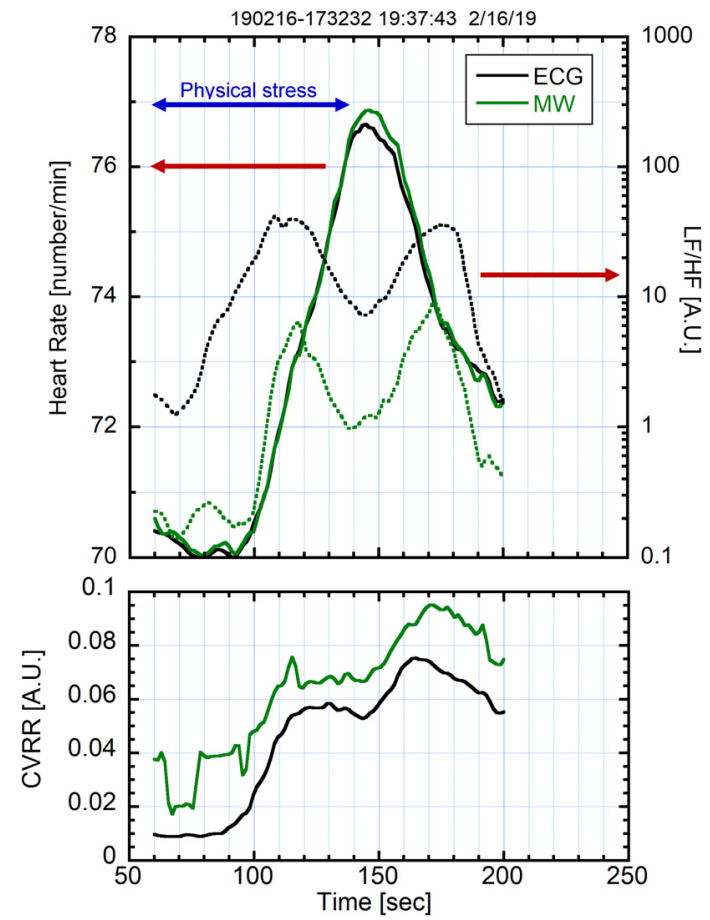

FIG. 10. Measurement of HR and HRV during artificial stress adding. The physical load is applied to a subject during 60-140 sec. 
As shown in Figs. 9 and 10, the value of LF/HF shows discrepancy between microwave and ECG although the tendency of time behavior agrees well. In the previous experiment using $10 \mathrm{GHz}$ reflectometer system, the value of $\mathrm{LF} / \mathrm{HF}$ has the similarity within $\pm 30 \%$ or more. However, the $24 \mathrm{GHz}$ system introduce much more discrepancy. It will be caused by the large phase fluctuation in the high frequency range due to short wavelength. Also the measurement of nonstatic subject introduce large high frequency noise.

Figure 11 shows time evolution of LF and HF components corresponding to Fig. 10. Note that the LF components agrees
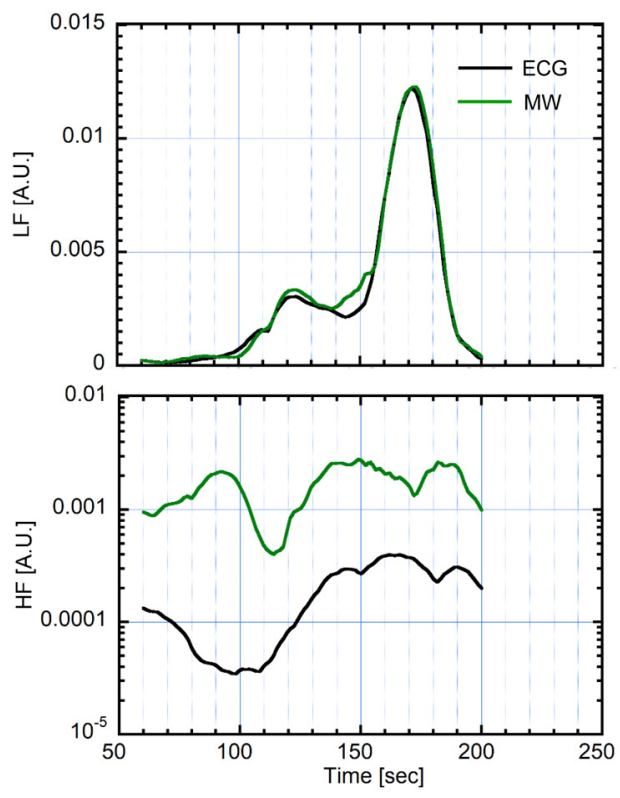

FIG. 11. Time evolution of LF and HF components corresponding to Fig. 10.

quite well between microwave and ECG measurements. On the other hand, the HF components shows large discrepancy, however, the time behaviors show some similarity.

In the present experiment (study protocol), subjects are not administered not to talk, but do not usually talk during the measurement. When a subject is talking, the reflectometer signal includes much more spurious components which will disturb the extraction of HR signal. The present measurements are limited to a subject working using a PC and driving a car. Therefore, we may suppose that a subject is usually not talking.

We can conclude that the $24 \mathrm{GHz}$ system is still useful for evaluation of human state by accumulating the data for various subjects. This is quite important since outdoor use is authorized in Japan as a movement body detection sensor for $24 \mathrm{GHz}$ radar system.

\section{CONCLUSIONS}

A radar reflectometer is applied to the biological (vital) signal detection We have proposed new signal processing techniques as well as system improvements such as cross-correlation between two reflectometers and I/Q detection with AGC amplifier. The instantaneous heart rate and heart-rate variability is measured and evaluated by using two types of algorithms (template/cross-correlation method and maximum entropy method). The values are in good agreement with those obtained by an ECG.

By combining microwave reflectometry and the proposed algorithms, the time behaviors of HR and HRV indices (LF, HF, and CVRR) can be obtained in real time. This potential will become useful for evaluation of human mental and physical condition in various environment such as under sleeping, working, and driving as well as to prevent sudden death related to coronary heart disease and ventricular arrhythmia.

In the present paper, the usefulness of microwave reflectometers for non-contact and non-invasive HR and HRV measurements is described. However, there still exist important issues. As a technical point, the influence of the spurious components due to conversation of subjects as well as the influence of the respiration, specifically, tachypnea. In a physics point of view, the clinical usefulness of the HR and HRV measurement is still an important issue. We will leave this for the future study.

\section{ACKNOWLEDGEMENTS}

This work is supported by the Grant-in Aid for Scientific Research, The Ministry of Education, Science, Sports, and Culture, Japan (No. 17K01310) and Adaptable and Seamless Technology Transfer Program through Target-Driven R\&D, Japan Science and Technology (No.AS2311355F). It is also performed with the support and under the auspices of the NIFS Collaboration Research program (NIFS17KKGP035, NIFS18KLEP027).

\section{NOMENCLATURE}

The acronyms utilized in the manuscript are summarized

CVRR Coefficient of variation of R-R intervals

ECG Electrocardiograph: the ECG recorder is modified to obtain the voltage output of the ECG waveform.

FFT Fast Fourier transform

HF High frequency: The frequency component of $0.15-$ $0.45 \mathrm{~Hz}$ in the HRV spectrum.

HR Heart rate

HRV Heart rate variability

LF Low frequency: The frequency component of 0.03$0.15 \mathrm{~Hz}$ in the HRV spectrum.

MEM Maximum entropy method

\section{REFERENCES}

${ }^{1}$ B. A. Williams, A. M. Honushefsky, and P. B. Berger, Am. J. Cardiol. 120, 1961 (2017).

${ }^{2}$ A. D. Lopez and T. Adair, Int. J. Epidemiology 48, ,"

${ }^{3}$ F. Sessa, V. Anna, G. Messina, G. Cibelli, V. Monda, G. Marsala, M. Ruberto, A. Biondi, O. Cascio, G. Bertozzi, D. Pisanelli, F. Maglietta, A. Messina, M. P. Mollica, and M. Salerno, Aging 10, 166 (2018).

${ }^{4}$ S. Hillebrand, K. B. Gast, R. de Mutsert, C. A. Swenne, J. W. Jukema, S. Middeldorp, F. R. Rosendaal, and O. M. Dekkers, EP Europace 15, 742 (2013).

${ }^{5}$ Task Force of The European Society of Cardiology and The North American Society of Pacing and Electrophysiology, European Heart Journal 17, 354 (1996).

${ }^{6}$ R.Sassi, S. Cerutti, F. Lombardi, M. Malik, H. Huikuri, C-K. Peng, G. Schmidt, and Y. Yamamoto, Europace 17, 1341 (2015).

${ }^{7}$ D. Zhang, X. Shen, and X. Qi, CMAJ 188, E53 (2016). 
${ }^{8}$ N. Brasier, C. J. Raichle, M. Dörr, A. Becke, V. Nohturfft, S. Weber, F. Bulacher, L. Salomon, T. Noah, R. Birkemeyer, and J. Eckstein, Europace 21, 41 (2019).

${ }_{9}^{9}$ J. C. Lin, Proc. IEEE 63, 1530 (1975).

${ }^{10}$ P. C. Pedersen, C. C. Johnson, C. H. Durney, and D. G. Bragg, IEEE Trans. Biomed. Eng. 23, 410 (1976).

${ }^{11}$ D. W. Griffin, Microwave J. 21, 69 (1978).

12 J. C. Lin, J. Kiernicki, M. Kiernicki, P. B. Wollschlaeger, IEEE Trans. Microw. Theory Tech. 27, 618 (1979).

${ }^{13}$ K.-M. Chen, D. Misra, H. Wang, H.-R. Chuang, E. Postow, IEEE Trans. Biomed. Eng. 33, 697 (1986).

${ }^{14}$ K.-M. Chen, Y. Huang, J. Zhang, and A. Norman, IEEE Trans. Biomed. Eng. 47, $105(2000)$

${ }^{15}$ Task Force of the European Society of Cardiology and the North American Society of Pacing and Electrophysiology, Circulation 93, 1043 (1996).

${ }^{16}$ U. Wiklund, M. Akay, and U. Niklasson, IEEE Eng. Med. Biol. Mag. 16, 113 (1997).

${ }^{17}$ L. Duvnjaek, S. Vuckovic, N. Car, and Z. Metelko, J. Diabetes Complications 15, 314 (2001)

${ }^{18}$ E. Landenberger-Leo, Med. Pr. 37, 347 (1986)

${ }^{19}$ M. F. Hilton, R. A. Bates, K. R. Godfrey, M. J. Chappell, and R. M. Cayton, 9ed. Biol. Eng. Comput. 37, 760 (1999).

${ }^{20}$ K. C. Bilchick, B. Fetics, R. Djoukeng, S. G. Fisher, R. D. Fletcher, S. N. Singh, E. Nevo, E. and R. D. Berger, Am. J. Cardio. 90, 24 (2002)..

${ }^{21}$ M. Takada, T. Ebara, and Y. Sakaki, Health Eval. Promot. 35, 373 (2008).

${ }^{22}$ S. Suzuki, T. Matsui, H. Imuta, M. Uenoyama, H. Yura, M. Ishihara, and M. Kawakami, Med. Biol. Eng. Comput. 46, 709 (2008).

${ }^{23}$ J.-M. Lee, H. C. Kim, J. I. Kang, and I Suh, BMC Psychol. 2, 29 (2014).

${ }^{24}$ A. Zhang, J. T. Hughes, A. Brown, P. D. Lawton, A. Cass, W. Hoy, K. O’Dea, and L. J. Maple-Brown, BMC Cardiovasc Disord. 16, 36 (2016).

${ }^{25}$ H-G. Kim, E-J. Cheon, D-S. Bai, Y. H. Lee, and B-H. Koo, Psychiatry Investig. 15, 235 (2018).

${ }^{26}$ D. Nagae, and A. Mase, Rev. Sci. Instrum. 81, 094301 (2010)

${ }^{27}$ A. Mase, and D. Nagae, US Patent. 9186079 (Nov. 17, 2015).

${ }^{28}$ Ministry of Internal Affairs and Communications (MIC). The Radio Use Web Site. https://www.tele.soumu.go.jp/j/adm/system/ml/small/index.htm (2019)

${ }^{29}$ M. Wakayama, H. Ezaki, I. Arai, and T. Miwa, IEICE Technical Report SANE2005-3, 13-16 (2005). (in Japanese)

${ }^{30}$ A. Lazaro, D. Girbau, and R. Villarino, Progress in Electromagnetic Research 100, 265 (2010)

${ }^{31}$ A. Mase, N. Ito, M. Oda, Y. Komada, D. Nagae, D. Zhang, Y. Kogi, S. Tobimatsu, T. Maruyama, S. Shimazu, F. Sakata, F. Sakai, D. Kuwahara, T. Yoshinaga, T. Tokuzawa, Y. Nagayama, K. Kawahata, S. Yamaguchi, S. TsujiIio, C. W. Domier, N. C. Luhmann, Jr., and H. K. Park, J. Instrum. 7, C01089 (2012).

${ }^{32}$ A. Mase, Y. Kogi, D. Kuwahara, Y. Nagayama, N. Ito, T. Maruyama, H. Ikezi, X. Wang, M. Inutake,T. Tokuzawa, J. Kohagura, M. Yoshikawa, S. Shinohara, A. Suzuki, F. Sakai, M. Yamashika, B. J. Tobias, C. Muscatello, X. Ren, M. Chen, C. W. Domier, and N. C. Luhmann, Jr., Adv. Phys: X 3, 1472529 (2018). ${ }^{33}$ The details obtaining optimum model order and other parameters for the MEM calculation are described in the Appendix A of Ref. 26. 